\title{
Young African Americans' Communication with Family Members About COVID-19: Impact on Vaccination Intention and Implications for Health Communication Interventions
}

\author{
Diane B. Francis $^{1}$ (I) Nia Mason ${ }^{2} \cdot$ Aurora Occa $^{1}$ \\ Received: 21 April 2021 / Revised: 22 June 2021 / Accepted: 23 June 2021 / Published online: 16 July 2021 \\ (C) W. Montague Cobb-NMA Health Institute 2021
}

\begin{abstract}
Communication with family members about the COVID-19 vaccine may play an important role in vaccination decisions, especially among young people. This study examined the association between family communication about the COVID-19 vaccine and vaccination intention. Participants were Black/African Americans aged 18-30 years $(N=312)$ recruited through an online survey in June 2020. We assessed family communication, vaccine attitudes, perceived norms, outcome expectancies, and vaccination intention. More than half $(62 \%)$ of the participants had talked to family members about the vaccine. Females were more likely than males to have engaged in family communication $(63 \%$ vs. $59 \%)(p .>.05)$. Family communication, injunctive norms, and descriptive norms were significantly $(p .<.01)$ associated with vaccination intention. Family communication was the strongest predictor of intention.

Promoting discussions about vaccinations between young Black adults and their families may increase the likelihood of adopting positive vaccination beliefs and influencing vaccine decision-making.
\end{abstract}

Keywords Family communication · COVID-19 · Vaccination · Black Americans

The COVID-19 pandemic has had a disproportionate impact on racial and ethnic minorities, including Black Americans. Racial and ethnic minorities are more likely to be infected with the virus, experience more severe illnesses, have higher rates of hospitalizations, and are at greater risk of death from COVID-19 [1-5]. And although young people are less likely to be hospitalized or die from coronavirus, they are just as at risk and have been severely impacted [6]. In fact, young people from racial and ethnic minority backgrounds have been burdened by the pandemic's social and economic costs.

COVID-19 vaccination is one strategy to mitigate the impact of the pandemic. As of March 2021, three vaccines have been granted emergency use authorization by the US Food and Drug Administration. The Advisory Committee on Immunization Practices (ACIP), part of the US Centers for

Diane B. Francis

diane.francis@uky.edu

1 Department of Communication, University of Kentucky, 274 Blazer Dining, 343 S Martin Luther King, Lexington, KY 40506, USA

2 Department of Mass Communication, Xavier University of Louisiana, New Orleans, LA, USA
Disease Control and Prevention (CDC), recommends a multiphase approach to distributing the vaccines. Per ACIP guidelines, the COVID-19 vaccine should be distributed last to persons aged 16 and older not included in one of the early priority groups [7]. The percentage of US adults who say they intend to get the COVID-19 vaccine when it is available to them has been steadily increasing since distribution began, with an average of about $70 \%$ of those polled willing to get the vaccine $[8,9]$. Yet, a large percentage of the public remains hesitant or skeptical of the vaccine. Furthermore, Black adults and young people are less willing to get the COVID-19 vaccine [8, 9]. Before the COVID-19 pandemic, Black Americans were less likely to get the flu vaccine than other groups [10]. Black women aged 19-26 were also less likely to have received the HPV vaccine [11]. While scant evidence exists on COVID-19 vaccine intention among young Black adults, an intersectional examination of the data suggests that they may be among those less willing to get vaccinated.

Empirical evidence suggests that family members play an essential role in health decision-making, including vaccination decisions. Parent-child communication about vaccines is a strong predictor of vaccine intention and behavior [12, 13]. However, there are few studies on the impact of family communication on vaccine intention and uptake among young 
adults. Even fewer studies have been conducted with underserved populations with lower vaccination rates, such as young Black men and women. Some studies have examined family communication and behavioral intentions and behavior among African Americans. For example, one study examined the association between African Americans' family communication and their willingness to donate organs [14], drawing on prior theorization about organ donation processes and the theory of reasoned action [15]. They found that individuals who had engaged in family discussions about organ donation had higher levels of knowledge, more favorable attitudes, and positive social norms [14]. The organ donation research centralized the role of family communication in the health decision-making process of African Americans [16, 17]. However, the research has rarely been extended to other health domains.

The purpose of the current study was to examine the association between family communication and COVID-19 vaccination intention among a sample of young Black adults. We argue that family members' opinions about the COVID-19 vaccine may play an important role in vaccination decisions, especially among young Black men and women. We focused on younger Black people because of existing vaccine gaps in this population. We posed one research question: What is the association between family communication and COVID-19 vaccination intention among young African American adults when accounting for these other factors? We further hypothesized that individuals who talked with family members about the vaccine would be more willing to get the vaccine; having discussions about the vaccine would be associated with more favorable vaccine attitudes, outcome expectancies, and perceived norms.

\section{Method}

\section{Participants and Procedures}

We recruited a sample of individuals from an online panel maintained by Qualtrics, a research organization. Data collection occurred in July 2020, months before the approval of the first vaccines for COVID-19 in the USA. To be eligible for the study, panel members had to self-identify as Black or African Americans and were aged 18-30 years old. A total of 312 participants completed the study.

Prospective participants were contacted via email by Qualtrics and asked to participate in a research study about the COVID-19 pandemic. After consenting to the study, participants answered a series of questions on their demographic characteristics, communication behaviors, attitudes, perceived norms, outcome expectancies, and intentions related to the vaccine. The questionnaire took about $10 \mathrm{~min}$ to complete.
The Institutional Review Board approved all study procedures at the University of Kentucky (IRB number: 58719).

\section{Measures}

The measures were adapted from previous research on interpersonal health communication [18], vaccination attitudes [19], and psychosocial determinants of behavioral intention (i.e., intention, injunctive and descriptive norms, outcome expectancies) [15]. ${ }^{1}$

Family Communication The primary predictor variable was family communication about the vaccine. Family communication about the vaccine was measured with one item [18]. We asked participants if they had ever talked with their family members about the COVID-19 vaccine (yes or no). We grouped the responses into "family talkers" and "non-talkers" [14]. We also asked follow-up questions to gain nuance on other discussion domains. We asked participants whether they had talked with family members about the pandemic, who did they talk to, and what else they talked about (i.e., family health history, end-of-life care, organ donation).

Vaccine Intention The primary outcome variable was intention to vaccinate against coronavirus. We assessed vaccination intention with a single item. Participants were given the statement, "I intend to get the coronavirus vaccine if a vaccine becomes available," and asked to respond $(0=$ No; $1=$ Yes $)$.

Covariates We assessed several covariates of vaccination intention, including attitudes, perceived norms (descriptive and injunctive), and outcome expectancies (positive and negative).

Vaccine attitudes were measured with the validated vaccine attitude examination scale [19]. Reliability on the 12item scale was good $(\alpha=.84)$. For our analyses, high scores indicate more favorable attitudes towards vaccines.

Injunctive norms were assessed by asking whether people agreed or disagreed that "A lot of people who are important to me think that getting the coronavirus vaccine would be a good thing." Descriptive norms were assessed by asking whether people agreed or disagreed that "It is expected that I would get the coronavirus vaccine if it became available." Both items were measured on a five-point scale from $1=$ strongly disagree to $5=$ strongly agree. We assessed both positive and negative outcome expectancies about getting the vaccine.

For positive outcome expectancies, participants were asked, "Which of the following are reasons you would get the coronavirus vaccine?" The 10 responses participants could select included items such as "I want to protect myself" and "I want to protect my family." For negative outcome

\footnotetext{
${ }^{1}$ Full details of measures used in the study, including the questionnaire and measures, are available from the first author.
} 
expectancies, participants were asked, "Which of the following reasons you would NOT get the coronavirus vaccine?" The nine responses participants could select included, "I would be concerned about side effects from the vaccine," "I would be concerned about getting infected with the coronavirus from the vaccine," and "I don't like needles." The expectancies were assessed on a four-point scale from strongly disagree to strongly agree. On a four-point scale, higher numbers indicate more positive and more negative outcome expectancies. The scale had good reliability for both the positive $(\alpha=$ $.89)$ and negative $(\alpha=.70)$ scales, respectively.

We also assessed a range of demographic variables, including age and sex.

\section{Results}

\section{Participant Characteristics}

Participant characteristics are reported in Table 1. The mean age of the sample was $22.67(S D=4.00)$. Most participants were non-Hispanic (82\%) and women (80\%). About half lived with a parent/guardian (49\%) and a quarter lived with a significant other $(23 \%)$. About a quarter of the sample (27\%) was unemployed, not in school; half were employed either part time $(22 \%)$ or full time $(31 \%)$. Forty-two percent of the sample had a high school diploma or equivalent degree, and a third (34\%) had a 4-year college degree or higher.

Almost all of the participants (95\%) had talked to family members about the pandemic. More than half (62\%) talked specifically about the vaccine. Females were more likely than males to have family discussions about coronavirus $(55 \% \%$ vs. $45 \%)$ and the vaccine ( $63 \%$ vs. $59 \%$ ) ( $p$ s. > .05). In addition to the vaccine, participation also had conversations about family health history (71\%), organ donation (61\%), and endof-life care (54\%). The majority (90\%) spoke to parents, $51 \%$ spoke to grandparents, and $71 \%$ spoke to other family members (e.g., siblings and cousins).

\section{Family Communication, Beliefs, and Vaccination Intention}

We used Pearson's chi-square analysis to examine differences in vaccination intention among those who had and had not discussed the vaccine. The analysis showed significant differences in vaccination intention among the two groups (Table 2): $62 \%$ of those who talked about the vaccine intended to vaccinate against COVID-19 compared to $44 \%$ of nontalkers, $\left.\chi^{2}(1,312)=12.59, p<.001\right)$. The hypothesis was supported.

Table 3 shows means, standard deviations, and correlations for all variables used in multivariate analyses. We hypothesized that individuals who talked with family members about
Table 1 Participant characteristics

\begin{tabular}{|c|c|c|}
\hline Variable & $n$ & $\%$ \\
\hline Age (M years; SD) & $22.67(4.00)$ & \\
\hline \multicolumn{3}{|l|}{ Gender } \\
\hline Female & 248 & 79 \\
\hline Male & 64 & 21 \\
\hline \multicolumn{3}{|l|}{ Ethnicity } \\
\hline Hispanic/Latino(a) & 57 & 18 \\
\hline Non-Hispanic/Latino & 255 & 82 \\
\hline \multicolumn{3}{|l|}{ Education } \\
\hline No high school or GED & 17 & 6 \\
\hline High school or GED & 131 & 42 \\
\hline 2-year college & 59 & 19 \\
\hline 4-year college & 65 & 21 \\
\hline Some graduate school & 10 & 3 \\
\hline Master's degree & 23 & 7 \\
\hline Advanced graduate degree & 7 & 2 \\
\hline \multicolumn{3}{|l|}{ Household income } \\
\hline Under $\$ 20,000$ & 109 & 35 \\
\hline$\$ 20,000-\$ 39,999$ & 60 & 19 \\
\hline$\$ 40,000-\$ 59,999$ & 59 & 19 \\
\hline$\$ 60,000-\$ 79,999$ & 34 & 11 \\
\hline$\$ 80,000-\$ 99,000$ & 14 & 4 \\
\hline$\$ 100,000-\$ 149,999$ & 28 & 9 \\
\hline$>\$ 150,000$ & 9 & 3 \\
\hline \multicolumn{3}{|l|}{ Marital status } \\
\hline Unmarried & 237 & 76 \\
\hline Unmarried, living with a partner & 35 & 11 \\
\hline Married & 33 & 10 \\
\hline Separated & 5 & 2 \\
\hline Widowed & 2 & 1 \\
\hline \multicolumn{3}{|l|}{ Residence } \\
\hline Live alone & 58 & 19 \\
\hline With parent/guardian & 152 & 49 \\
\hline With significant other & 73 & 23 \\
\hline Other & 29 & 9 \\
\hline
\end{tabular}

the vaccine would be more willing to get the vaccine. We also predicted that individuals who had family discussions about the vaccine would report more favorable attitudes, outcome
Table 2 COVID-19 vaccine intention by family communication group

\begin{tabular}{lll}
\hline Group & \multicolumn{2}{l}{ Vaccine Intention } \\
\cline { 2 - 3 } & Yes (n, \%) & No (n, \%) \\
\hline Talkers & $106(64)$ & $59(36)$ \\
Non-talkers & $65(44)$ & $82(56)$ \\
\hline$\chi^{2}(1)=12.59, p<.001$ &
\end{tabular}


Table 3 Means, standard deviations, and correlation matrix for variables in the study

\begin{tabular}{llllllllll}
\hline & $M$ & $S D$ & 1 & 2 & 3 & 4 & 5 & 6 & 7 \\
\hline 1. Family communication & - & - & - & & & & & & \\
2. Vaccination attitudes & 2.84 & .74 & -.02 & - & & & & & \\
3. Injunctive norms & 3.42 & 1.35 & $.17^{* *}$ & $.29^{* *}$ & - & & & \\
$\begin{array}{l}\text { 4. Descriptive norms } \\
\text { 5. Positive outcome }\end{array}$ & 3.38 & 1.38 & .06 & $.31^{* *}$ & $.73^{* *}$ & - & & \\
$\quad$ expectancies & 2.89 & .77 & $.17^{* *}$ & $.15^{* *}$ & $.37^{* *}$ & $.42^{* *}$ & - & & \\
$\begin{array}{l}\text { 6. Negative outcome } \\
\quad \text { expectancies }\end{array}$ & 2.25 & .61 & .02 & $-.30^{* *}$ & $-.29^{* *}$ & $-.31^{* *}$ & -.09 & - & \\
7. Vaccine intention & - & - & $.20^{* *}$ & $.25^{* *}$ & $.53^{* *}$ & $.59^{* *}$ & $.35^{* *}$ & $-.19^{* *}$ & - \\
\hline
\end{tabular}

$* * \mathrm{p}<.01$

expectancies, and perceived norms. We conducted a series of one-way ANOVAS to test this hypothesis. The analysis compared talkers with non-talkers (Table 4). We did not find any statistical differences in vaccination attitudes among those who had family discussions $(M=2.83, S D=.76)$ compared to those who did not have such discussions $(M=2.86, S D=$ $.72), F(1,311)=.17, p=.68$. However, individuals who had talked about the vaccine had more favorable positive outcome expectancies $(M=3.02, S D=.78)$ than individuals who did not have such discussions $(M=2.76, S D=.72), F(1,311)=$ $9.10, p<.01$. There were no differences in negative outcome expectancies among the two groups $\left(M_{\text {talkers }}=2.26, S D=.60\right.$; $\left.M_{\text {nontalkers }}=2.24, S D=.62\right), F(1,311)=.08, p=.78$. A similar pattern emerged for perceived norms. Individuals who had talked about the vaccine had more favorable injunctive norms $(M=3.62, S D=1.27)$ than individuals who did not have such discussions $(\mathrm{M}=3.18, \mathrm{SD}=1.30), F(1,311)=8.94, p<.01$. However, there were no differences in descriptive norms $\left(M_{\text {talkers }}=3.46, S D=1.40 ; M_{\text {nontalkers }}=3.29, S D=1.16\right)$, $F(1,311)=.08, p=.28$ Thus, individuals who talked to family members about the vaccine had more favorable injunctive norms and higher positive outcome expectancies scores. The hypothesis was partially supported.

This study's research question concerned the role of family communication on vaccination intention when accounting for factors such as attitudes, outcome expectancies, and perceived norms. We conducted a logistic regression analysis to examine the association between these variables. All variables, as well as age and sex, were entered into the model simultaneously (Table 5). Family communication about the vaccine $(p=.005)$, injunctive norms $(p=.004)$, and descriptive norms $(p<.001)$ were significantly associated with vaccination intention. Vaccination attitudes $(p=.10)$ and positive outcome expectancies $(p=.07)$ were not associated with vaccination intention. Family communication was the strongest predictor of vaccination intention. Individuals who engaged in family discussions about the vaccine were more than twice as likely to intend to get vaccinated compared to those who did not discuss the vaccine with family members. The model predicted between 38 and $51 \%$ of the variance in vaccination intention based on Cox and Snell's $\mathrm{R}^{2}$ and Nagelkerke's $\mathrm{R}^{2}$ values, respectively.

\section{Discussion}

The COVID-19 pandemic has been a topic of much discussion among American adults, including many Black people. A survey by Pew Research Center in April 2020 showed that Black adults were more likely than white adults to talk about the virus outbreak; $53 \%$ of Black adults frequently discussed the COVID-19 pandemic compared with $44 \%$ of US adults generally [20]. Beyond general conversations about the pandemic, it remains an empirical question whether discussions that are specifically about the vaccine influence downstream COVID-19 vaccine uptake. This study provides evidence that
Table 4 Means, standard deviations, and one-way analyses of variance by family communication type

\begin{tabular}{|c|c|c|c|c|c|c|}
\hline & \multicolumn{2}{|c|}{ Talkers } & \multicolumn{2}{|c|}{ Non-talkers } & \multirow[t]{2}{*}{$F(1,311)$} & \multirow[t]{2}{*}{$\eta^{2}$} \\
\hline & $M$ & $S D$ & $M$ & $S D$ & & \\
\hline Vaccination attitudes & 2.83 & .76 & 2.86 & .72 & .17 & .001 \\
\hline Injunctive norms & 3.62 & 1.27 & 3.18 & 1.30 & $8.94 * *$ & .028 \\
\hline Descriptive norms & 3.46 & 1.40 & 3.29 & 1.36 & 1.16 & .004 \\
\hline Positive outcome expectancies & 3.02 & .78 & 2.76 & .72 & $9.10 * *$ & .029 \\
\hline Negative outcome expectancies & 2.26 & .60 & 2.24 & .62 & .08 & .001 \\
\hline
\end{tabular}

$* * \mathrm{p}<.01$ 
Table 5 Multivariate logistic regression predicting vaccine intention

\begin{tabular}{lllll}
\hline Variable & $B$ & $S E$ & $p$ & $95 \%$ CI \\
\hline Family communication & 2.36 & .31 & .005 & {$[1.29,4.31]$} \\
Vaccination attitudes & 1.40 & .24 & .095 & {$[.93,2.42]$} \\
Injunctive norms & 1.57 & .16 & .004 & {$[1.16,2.13]$} \\
Descriptive norms & 2.28 & .16 & .001 & {$[1.65,3.14]$} \\
Positive outcome expectancies & 1.52 & .23 & .073 & {$[.96,2.35]$} \\
Negative outcome expectancies & 1.04 & .29 & .905 & {$[.59,1.83]$} \\
\hline
\end{tabular}

Note: $C I$ confidence interval

family communication, at least among the Pew sample of young African American adults, may be critical to their COVID-19 vaccine decision-making process.

We found that family communication about the COVID19 vaccine significantly predicted vaccine intention among young adults. Furthermore, family communication was the strongest predictor of vaccination intention above and beyond other known predictors such as attitudes and perceived norms. Our findings for family communication are in line with previous studies examining family conversations in different contexts, such as organ donation [17, 21]. However, unlike other studies, attitudes and expectancies did not have a significant role in this study. A possible explanation is that the vaccine was not yet available when data were collected, making outcome expectancies only speculative rather than information-based. Nevertheless, family communication plays a vital role in several health behaviors. While many adults ultimately make vaccination decisions independently, some do make decisions collectively with their family members. Some research indicates that health discussions encourage cognitive elaboration and other forms of information processing [22]. In turn, elaboration is associated with positive behavior change [22]. Through discussions, others can be persuaded toward an acceptance (reduced hesitancy) of the COVID-19 vaccine.

These findings can support public health experts' and campaign planners' decision-making when developing strategies to improve COVID-19 vaccination rates among young African Americans. While many resources will be devoted to the deployment of campaigns to increase vaccination rates across the USA, some effort should be spent understanding the variables that affect willingness to talk about the vaccine and be directed to encourage young Black men and women to talk with family members about the vaccine and their decisions to vaccinate. Younger people may rely more on the vaccine experiences of those closer in their networks (e.g., family members) than others in the public (e.g., celebrities or public figures) when making decisions about the COVID19 vaccine. Motivating younger people to be active participants in the vaccination process will be vital to achieving optimal vaccination rates.
In our study, vaccine attitudes were not associated with vaccine intention; conversely, family communication was associated with vaccine intention. The findings for family communication could be because a very high percentage of the sample had communicated with family members about the virus and the vaccine. The findings for vaccine attitudes contradict other research on the relation between attitudes and intentions. This could result from how vaccine attitudes were measured [19]; the vaccine attitude examination scale may not have captured attitudes related to the COVID-19 vaccine, which can be explored in future research. That said, some research and theorizing on determinants of intentions suggest that intentions could be driven by attitudes, norms, self-efficacy, or other variables [15]. Thus, it is possible that for the COVID-19 vaccine, other determinants could and should be examined, as found in this study.

\section{Limitations and Future Directions}

There are some limitations of this study that should be acknowledged. First, we used a convenience sample of men and women in a specific age range. We recruited participants with varying educational and employment statuses; however, the sample was majority female. Females in our sample were more likely to talk than males. However, the findings were not statistically significant, potentially due to the smaller number of males in the study. But the high percentage of females in the sample could be a likely explanation for the main findings of the association between family conversations and vaccine intention. Prior research suggests that young Black women's communication with family and friends is associated with behavioral intentions [18]. The research with young Black men is less known but would add to understanding the relation between family conversations and vaccine intention and behaviors. Second, given our sample's characteristics, caution must be taken when trying to generalize to younger Black individuals or even all Black Americans. There are very few studies of Black Americans' communication with family members about vaccination and its impact. Our study contributes to this gap in the research. Future studies should seek to replicate these findings with a more diverse sample of Black Americans and further assess within-group differences.

Third, while our study focused on the presence of family communication about the COVID-19 vaccine, we did not investigate the valence, tone, or quality of such conversations. We also did not seek to identify what happens in the absence of such conversations. But the critical role of family discussions on individuals' health decision-making is evident when such discussions were missing. This is especially the case of topics generally considered "taboo" $[18,23]$. We also did not investigate what influences family communication, such as media exposure to information about the vaccine. The literature also indicates that the media affect family discussions 
about health in several ways. For example, in organ donation research, media information was used to inform the beliefs shared during family discussions [24]. Each of these areas presents opportunities for continued research on family communication about COVID-19 among young African Americans.

It should be noted that family conversations could encourage or discourage COVID-19 vaccination. Family conversations could also be a source of misinformation about the vaccines. Promoting positive discussions about vaccinations between young Black men and women and their family members may increase the likelihood of adopting positive COVID19 vaccination beliefs and may influence vaccine decisionmaking. Conversely, if such conversations discourage vaccinations or introduce misinformation, it is essential to develop or reinforce skills such as saying something as soon as the misinformation is introduced [25].

Future studies should also evaluate message strategies based on the findings from this study. For example, considering the critical role of family discussions, messages for COVID-19 vaccination educational interventions could provide examples of arguments to initiate family discussions and evidence and strategies to support them. In addition, considering the effects observed for injunctive norms, future campaigns could focus on people's perceptions of Black Americans' vaccination behaviors. A strategy could be to provide real testimonials and experiences of young Black Americans receiving the vaccine. Testimonials, if done well, usually generate interpersonal communication within family and friendship networks. Such conversations, in turn, can generate behavior change.

\section{Conclusion}

Optimal vaccination among young people, including those from racial and ethnic minority groups, is critical to achieving equitable distribution of the vaccine and mitigating the persistent disparities experienced by these communities. This is the first study, to our knowledge, to assess the association between family communication and vaccination intention against COVID-19 among a sample of young African American adults. This study adds to research aiming to advance knowledge of family communication patterns about the pandemic and its impacts on health decision-making and health behaviors. Advancing knowledge about health-related family communication among young African American adults may aid in developing family- and network-based interventions with the potential to motivate vaccination disparities.

\section{Availability of Data and Material Not applicable}

\section{Declarations}

Ethics Approval All study procedures were approved by the Institutional Review Board at the University of Kentucky (IRB number: 58719).

Consent to Participate Consent was obtained verbally from all participants.

Consent for Publication All authors consent to the publication of this manuscript.

Competing Interests The authors declare no competing interests.

\section{References}

1. Gold JAW, Wong KK, Szablewski CM, Patel PR, Rossow J, da Silva J, et al. Characteristics and clinical outcomes of adult patients hospitalized with COVID-19 - Georgia, March 2020. MMWR Morb Mortal Wkly Rep. 2020;69:545-50. https://doi.org/10. 15585/mmwr.mm6918e1.

2. Stokes EK, Zambrano LD, Anderson KN, Marder EP, Raz KM, El Burai FS, et al. Coronavirus disease 2019 case surveillance - United States, January 22-May 30, 2020. MMWR Morb Mortal Wkly Rep. 2020;69:759-65. https://doi.org/10.15585/mmwr.mm6924e2.

3. Wortham JM, Lee JT, Althomsons S, Latash J, Davidson A, Guerra $\mathrm{K}$, et al. Characteristics of persons who died with COVID-19 United States, February 12-May 18, 2020. MMWR Morb Mortal Wkly Rep. 2020;69:923-9. https://doi.org/10.15585/mmwr. mm6928e1.

4. Moore JT, Ricaldi JN, Rose CE, Fuld J, Parise M, Kang GJ, et al. Disparities in incidence of COVID-19 among underrepresented racial/ethnic groups in counties identified as hotspots during June 5-18, 2020 - 22 States, February-June 2020. MMWR Morb Mortal Wkly Rep. 2020;69:1122-6. https://doi.org/10.15585/ mmwr.mm6933e1.

5. Garg S. Hospitalization rates and characteristics of patients hospitalized with laboratory-confirmed coronavirus disease 2019COVID-NET, 14 States, March 1-30, 2020. MMWR Morb Mortal Wkly Rep. 2020;69:458-64. https://doi.org/10.15585/ mmwr.mm6915e3.

6. Boehmer TK, DeVies J, Caruso E, van Santen KL, Tang S, Black $\mathrm{CL}$, et al. Changing age distribution of the COVID-19 pandemic United States, May-August 2020. MMWR Morb Mortal Wkly Rep. 2020;69:1404-9. https://doi.org/10.15585/mmwr.mm6939e1.

7. Dooling K, Marin M, Wallace M, McClung N, Chamberland M, Lee GM, et al. The advisory committee on immunization practices' updated interim recommendation for allocation of COVID-19 vaccine - United States, December 2020. MMWR Morb Mortal Wkly Rep. 2021;69:1657-60. https://doi.org/10.15585/mmwr. mm695152e2.

8. Hamel L, Kirzinger A, Lopes L, Kearney L, Sparks G, Brodie M. KFF COVID-19 vaccine monitor: January 2021. Kaiser Family Foundation, 2021. Available from: https://www.kff.org/ coronavirus-covid-19/report/kff-covid-19-vaccine-monitorjanuary-2021/.

9. Funk C, Tyson A. Growing share of Americans say they plan to get a COVID-19 vaccine - or already have: Pew Research Center; 2021. Available from: https://www.pewresearch.org/science/2021/ 03/05/growing-share-of-americans-say-they-plan-to-get-a-covid19-vaccine-or-already-have/. 
10. CDC flu vaccination coverage, United States 2018-2019 influenza season [Internet]. 2019. Available from: https://www.cdc.gov/flu/ fluvaxview/coverage-1819estimates.htm.

11. U.S. Department of Health and Human Services Office of Minority Health. Immunizations and African Americans. 2020. Available from: https://minorityhealth.hhs.gov/omh/browse.aspx?lvl= 4\&lvlid $=22$

12. McRee AL, Reiter PL, Gottlieb SL, Brewer NT. Mother-daughter communication about HPV vaccine. J Adolesc Health. 2011;48: 314-7. https://doi.org/10.1016/j.jadohealth.2010.07.006.

13. Hesse C, Rauscher EA. The relationship between family communication patterns and child vaccination intentions. Commun Res Rep. 2016;33:61-7. https://doi.org/10.1080/08824096.2015.1117444.

14. Morgan SE. The power of talk: African Americans' communication with family members about organ donation and its impact on the willingness to donate organs. J Soc Pers Relat. 2004;21:112-24. https://doi.org/10.1177/0265407504039845.

15. Fishbein M, Ajzen I. Predicting and changing behavior: the reasoned action approach: Psychology Press; 2010.

16. Morgan SE, Cannon T. African Americans' knowledge about organ donation: closing the gap with more effective persuasive message strategies. J Natl Med Assoc. 2003;95:1066-71.

17. Morgan SE, Miller JK, Arasaratnam LA. Similarities and differences between African Americans' and European Americans' attitudes, knowledge, and willingness to communicate about organ donation. J Appl Soc Psychol. 2003;33:693-715. https://doi.org/ 10.1111/j.1559-1816.2003.tb01920.x.

18. Francis DB, Zelaya CM, Fortune DA, Noar SM. Black college women's interpersonal communication in response to a sexual health intervention: a mixed methods study. Health Commun. 2021;36: 217-25. https://doi.org/10.1080/10410236.2019.1673949.

19. Martin LR, Petrie KJ. Understanding the dimensions of antivaccination attitudes: the vaccination attitudes examination (vax) scale. Ann Behav Med. 2017;51:652-60. https://doi.org/10.1007/ s12160-017-9888-y.
20. Mitchell A, Jurkowitz M. Black U.S. Adults follow many COVID19 news topics more closely, discuss the outbreak more frequently: Pew Research Center; 2020. Available from: https://www. pewresearch.org/fact-tank/2020/06/05/black-u-s-adults-followmany-covid-19-news-topics-more-closely-discuss-the-outbreakmore-frequently/.

21. Afifi WA, Morgan SE, Stephenson MT, Morse C, Harrison T, Reichert $\mathrm{T}$, et al. Examining the decision to talk with family about organ donation: applying the theory of motivated information management. Commun Monogr. 2006;73:188-215. https://doi.org/10. 1080/03637750600690700.

22. Eveland WP. The effect of political discussion in producing informed citizens: the roles of information, motivation, and elaboration. Polit Commun. 2004;21:177-93. https://doi.org/10.1080/ 10584600490443877

23. Stewart JL, Widman L, Kamke K. Applying a multifactorial communication framework to better understand differences between father-daughter and mother-daughter sexual health discussions. J Health Commun. 2019;24:633-42. https://doi.org/10.1080/ 10810730.2019.1651428.

24. Morgan SE, Harrison TR, Long SD, Afifi WA, Stephenson MS, Reichert T. Family discussions about organ donation: how the media influences opinions about donation decisions. Clin Transplant. 2005;19:674-82. https://doi.org/10.1111/j.1399-0012.2005.00407. $\mathrm{x}$.

25. Bode L, Vraga EK. See something, say something: correction of global health misinformation on social media. Health Commun. 2018;33:1131-40. https://doi.org/10.1080/10410236.2017. 1331312.

Publisher's Note Springer Nature remains neutral with regard to jurisdictional claims in published maps and institutional affiliations. 